\title{
DISEÑO ESPECULATIVO, CO-CREACION Y CASA DEL PUENTE. Un caso de prácticas didácticas en entorno post-digital en la carrera de Arquitectura
}

\author{
SPECULATIVE DESIGN, CO-CREATION AND CASA DEL PUENTE. A case of teaching \\ practices in post-digital environment in the career of Architecture
}

\author{
Dra. Arqta. Diana Rodríguez Barros \\ FAUD UNMdP, Argentina \\ dibarros@mdp.edu.ar
}

\author{
Dis. Graf. María Mandagarán \\ FAUD UNMdP, Argentina \\ mmandagaran@hotmail.com
}

\begin{abstract}
The creation can be considered as co-creation in both shared operations from interventions linked to the presence of the other. In virtual environments and interconnected in the Web, post-digital environments, these activities are provided in a unique way. Certain proyectual practices framed in Design Thinking, are stimulated by a proactive action in this direction. In particular, as in the case of Speculative Design, which explores implications of what is not reality yet, and links new technologies, scientific applications and aesthetic trends. In this direction we present teaching practices, next to Media-Labs modalities. They were realized by architecture students. They were focused in re-inserts, re-functionalizations and re-significations of the Casa del Puente, Mar del Plata, emblematic building of latin american modernism. The experience, with mostly positive result, was analyzed and evaluated according to co-creativity indicators. We conclude that these experiences rub the expressive and creative disciplinaries boundaries of Architecture career, and are close to transdisciplinar concept of Art Thinking.
\end{abstract}

Keywords: Didactic practice; Architecture; Speculative design; Co-creation; Bridge's house/Casa del Puente.

\section{Introducción}

La creación es factible de ser asumida como co-creación en tanto operaciones compartidas, pero a la manera de intervenciones vinculadas con la presencia de los otros. Tanto para generar, valorar y registrar acciones $y$ producciones, como para proporcionar opciones de recreación y reformulación de procesos y resultados. Según Sanders \& Simons (2009), todos podemos ser creativos pero además trabajar colaborativamente, aceptar la diversidad de posturas y criterios, interacturar entre los involucrados, tener capacidad de diálogo y generar construcción compartida.

En particular, en los entornos virtuales e interconectados de la Web, donde se superponen e hibridan prácticas propias de Web Estática 1.0, Web Social 2.0, Web Semántica 3.0 y Web Omnipresente 4.0, estas actividades se ven facilitadas de manera singular. Estos entornos mutantes, los entornos postdigitales, se caracterizan por estados en permanente fluidez y actualización, destacan sus rasgos virtuales con prevalencia visual y están indefectiblemente interconectados desde la ubicuidad (Pardo Kuklinsky, 2010; Lima, 2011).

Reconocemos que prácticas proyectuales vinculadas a determinadas prácticas de diseño, encuadradas desde la perspectiva del Pensamiento de Diseño (Brown, 2009), se ven estimuladas desde un accionar proactivo en esta dirección. Tal el caso de las modalidades propias del Diseño Especulativo, que interpretamos como propuestas abiertas, orientadas al debate y la polémica. $Y$ en simultáneo explorando nuevas implicaciones de lo que aún no es realidad, con niveles amplios de incertidumbre, así como vinculando nuevas tecnologías, aplicaciones científicas y tendencias estéticas (Dunne \& Raby, 2013).

Nos ha interesado indagar y experimentar en esta dirección desde un caso de intervención pedagógica. Específicamente lo hemos afrontado, basados en experiencias anteriores, (Rodríguez Barros y Mandagarán, 2014), enfocando momentos de gestión, diseño y producción de bases de datos gráficas interviniendo con obras paradigmáticas de Arquitectura, para generar nuevos contextos de reinserción y simulación hiperrealista.

En esta oportunidad nos orientamos hacia modalidades próximas a las prácticas de los ambientes de laboratorio Labs de Arte/Ciencia en entorno post-digital. Nos ha interesado profundizar el rol de las narrativas de base visual en procesos de enseñanza como recurso didáctico facilitador de construcción de sentido. Asumimos que esta modalidad de trabajo implica por un lado, ambientes de ideación, producción y difusión orientados hacia proyectos de investigación en la convergencia del diseño, la transmedialidad y la tecnología; por otro, explora formas de experimentación y aprendizaje colaborativo surgido de las redes digitales. De todas formas, reconocemos las limitaciones que tal práctica didáctica implica al respecto.

Complementariamente hemos observado (Rodríguez Barros, et.al. 2015) que el tratamiento y manipulación de la imagen digital aporta repertorios extensos de recursos técnicos con alto valor agregado en procesos proyectuales. En esta dirección, los entornos post-digitales ofrecen experiencias enriquecedoras, interactivas y ubicuas potenciadas por el 
uso de recursos gratuitos para generar y compartir información multi e hipermedial (Cramer, 2014).

Conjeturamos que tales experiencias, pueden ofrecer un aporte positivo y considerable en intervenciones propias de ámbitos proyectuales en relación con las tecnologías postdigitales.

\section{Marco conceptual}

El Pensamiento de Diseño, siguiendo a Tim Brown (2009, op.cit.), es una corriente que remite a procesos básicos referidos a empatía, creación colaborativa o co-creación, prototipado y sustento propio desde narrativas eminentemente de carácter visual. A partir de estos rasgos fundamenta y expresa estrategias metodológicas de diseño para tratar problemas complejos que afectan a colectivos amplios y diversos, que no se resuelven con soluciones simples, y que requieren la participación directa y activa de los futuros implicados. Reconoce deseos de los usuarios, factibilidad tecnológica, sustenibilidad y viabilidad económica. Aspira innovar desde el diseño en el devenir de experiencias que vinculen objetos, procesos, emociones y funciones. Resulta básico y necesario conocer y re-conocer a las personas para las que se diseña, formular respuestas inéditas, prototipar rápida y repetitivamente los diseños, y sostener argumentos para contar historia de modo atractivo y convincente que provoquen el interés, la comprensión y la inclusión.

En los ambiente post-digitales, integrados y reticulares, resultan fundacionales las narrativas visuales que les dan sentido y están próximas a ser soportadas por intervenciones transmediales, como sostiene Carlos Scolari (2013). Las mismas reflejan la creación de mundos (narrativos) donde se constituyen en experiencias innovadoras, relatos, medios expresivos y plataformas diversas. Coincidimos con Henry Jenkins (2012), cuando sostiene que tales narraciones transmedia storytelling' resultan las expresiones estéticas ideales en la era de la inteligencia colectiva y las intervenciones crowd de las multitudes.

En esta dirección, hemos observados prácticas proyectuales vinculadas al Diseño Especulativo que proponen contribuir al debate socio-cultural de la cultura visual contemporánea, afrontar lo desconocido y lo ambiguo de forma no convencional, así como transitar la hibridación entre diseño participativo y emergente. Se caracterizan por ser sustentables, rediseñables, reconfigurables y cambiantes. Asimismo posicionarse en interacción con objetos dilemáticos. Controversialmente proponen nuevas alternativas de creación y co-creación de objetos, sistemas originales y contradictorios de naturaleza tecno-cultural. De forma prioritaria aspiran crear no sólo cosas sino en particular ideas. Por lo tanto y como indican lbars \& Ma (2013), cuestionan, reformulan y probablemente sostienen modificaciones de comportamientos, pensamientos y acciones para un mundo que podría ser.

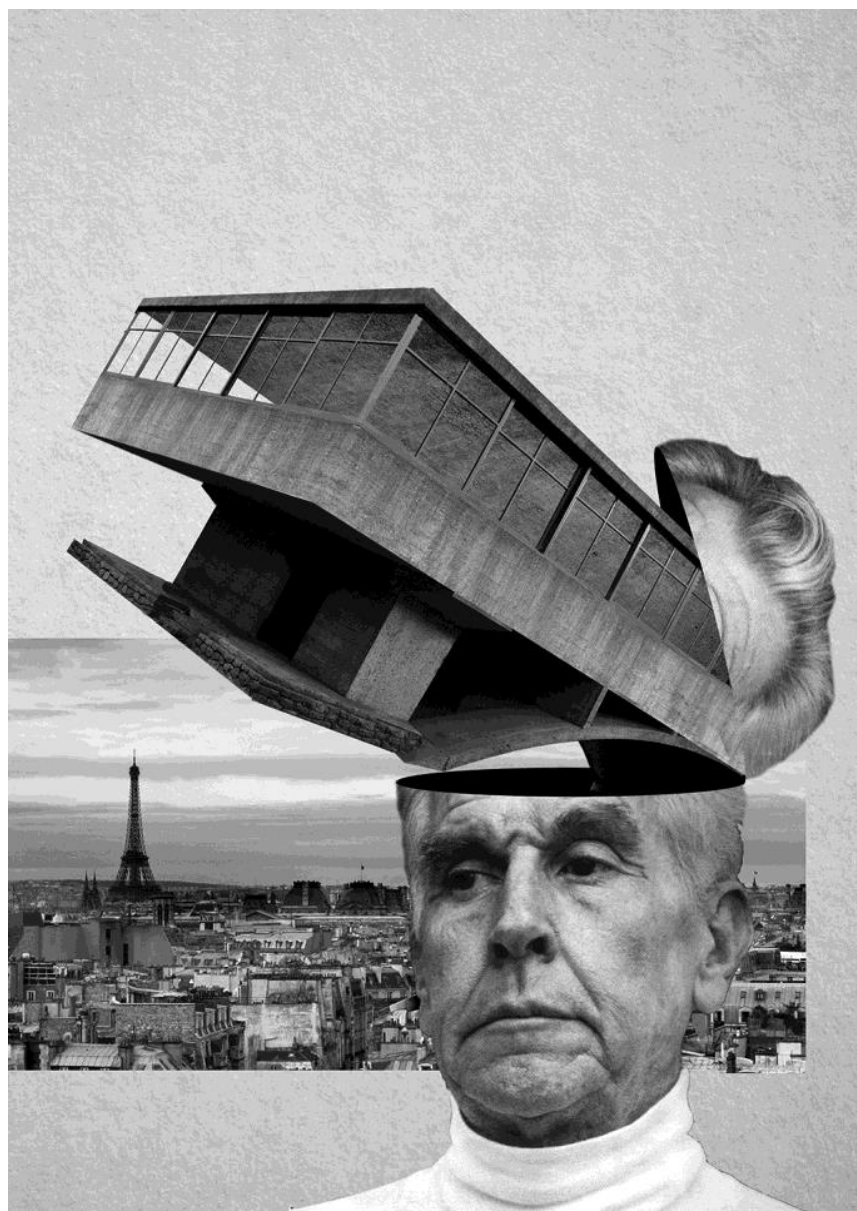

Figura 1. Arq. Williams y Casa del Puente. Gastón De Dios, 2015

\section{Presentación del caso}

Desde este encuadre conceptual, presentamos una práctica didáctica desarrollada en el curso Entornos post-digitales y fotomontajes. De duración cuatrimestral, fue realizada durante los ciclos lectivos 2015 y 2016, como asignatura electiva Area Arquitectónica Urbanística Ciclo Orientación, carrera de Arquitectura, Facultad Arquitectura, Urbanismo y Diseño, Universidad Nacional de Mar del Plata, Argentina.

Nos basamos en experiencias anteriores que realizó el grupo docente (Rodríguez Barros, et.al.2015 op.cit.; Rodríguez Barros, Mandagarán, 2014 op.cit.). La misma estuvo centrada en re-inserciones, re-funcionalizaciones y resignificaciones de una obra arquitectónica prestigiada en nuevos entornos, por un lado sustentables (naturales y urbanos) y por otro imaginarios.

En este caso, abordamos el tratamiento y manipulación de la imagen digital pixelar y vectorial, junto a los recursos técnicos de aplicaciones libres y colaborativas disponibles en la Web con alto valor agregado en los procesos proyectuales, en tanto facilitan el acceso a experiencias interactivas para generar y compartir información pregnante de naturaleza gráfica y móvil. Afrontamos así el planteo y desarrollo de narrativas visuales. Recurrimos centralmente a 
SIGraDi 2016, XX Congreso de la Sociedad Ibero-americana de Gráfica Digital 9-11, November, 2016 - Buenos Aires, Argentina

la creación de fotomontajes hiperrealistas, junto a infografías y piezas comunicacionales diversas como recursos

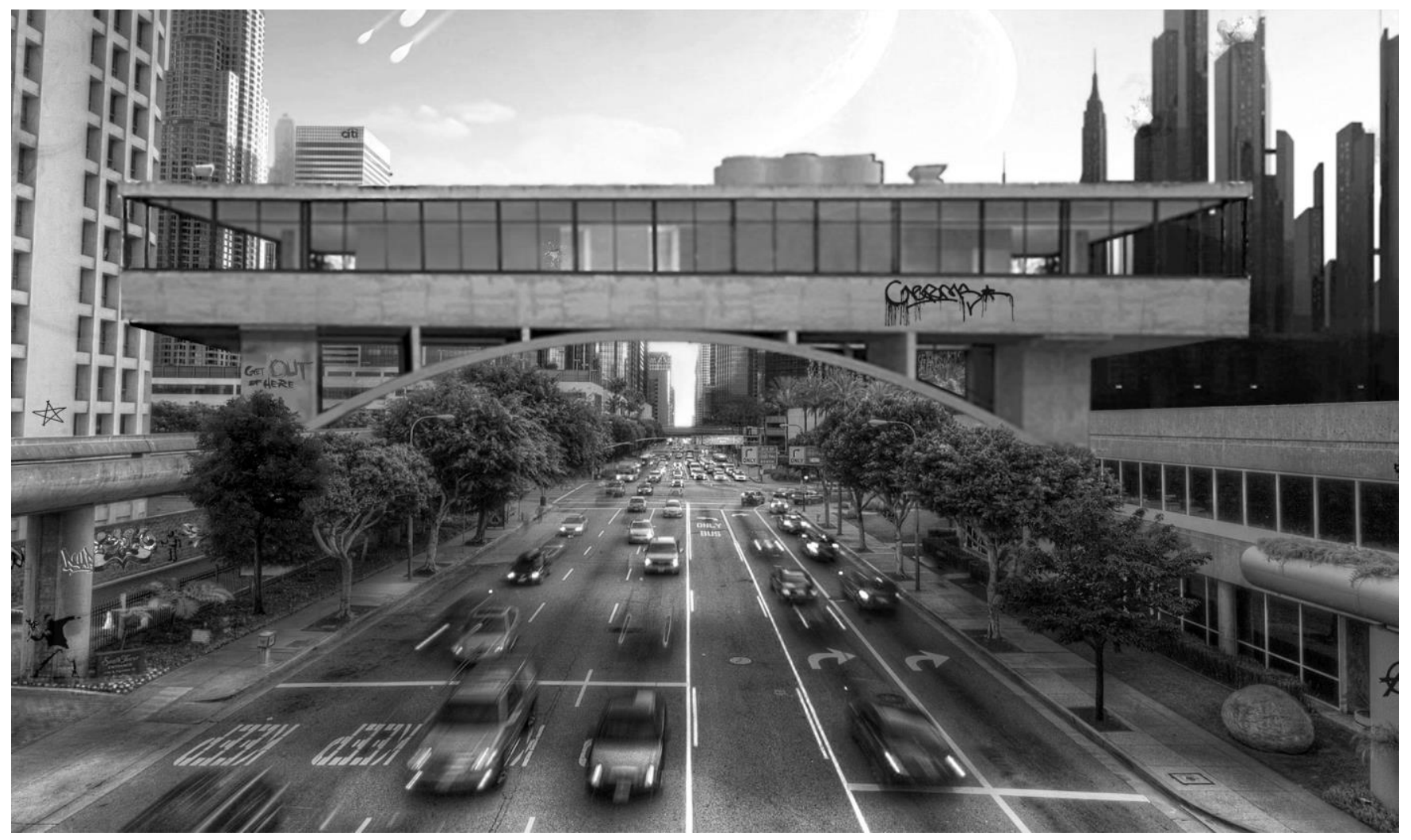

Figura 2. Fotomontaje exterior. Ezequiel Muñoz y Manuela Pozzobón, 2016

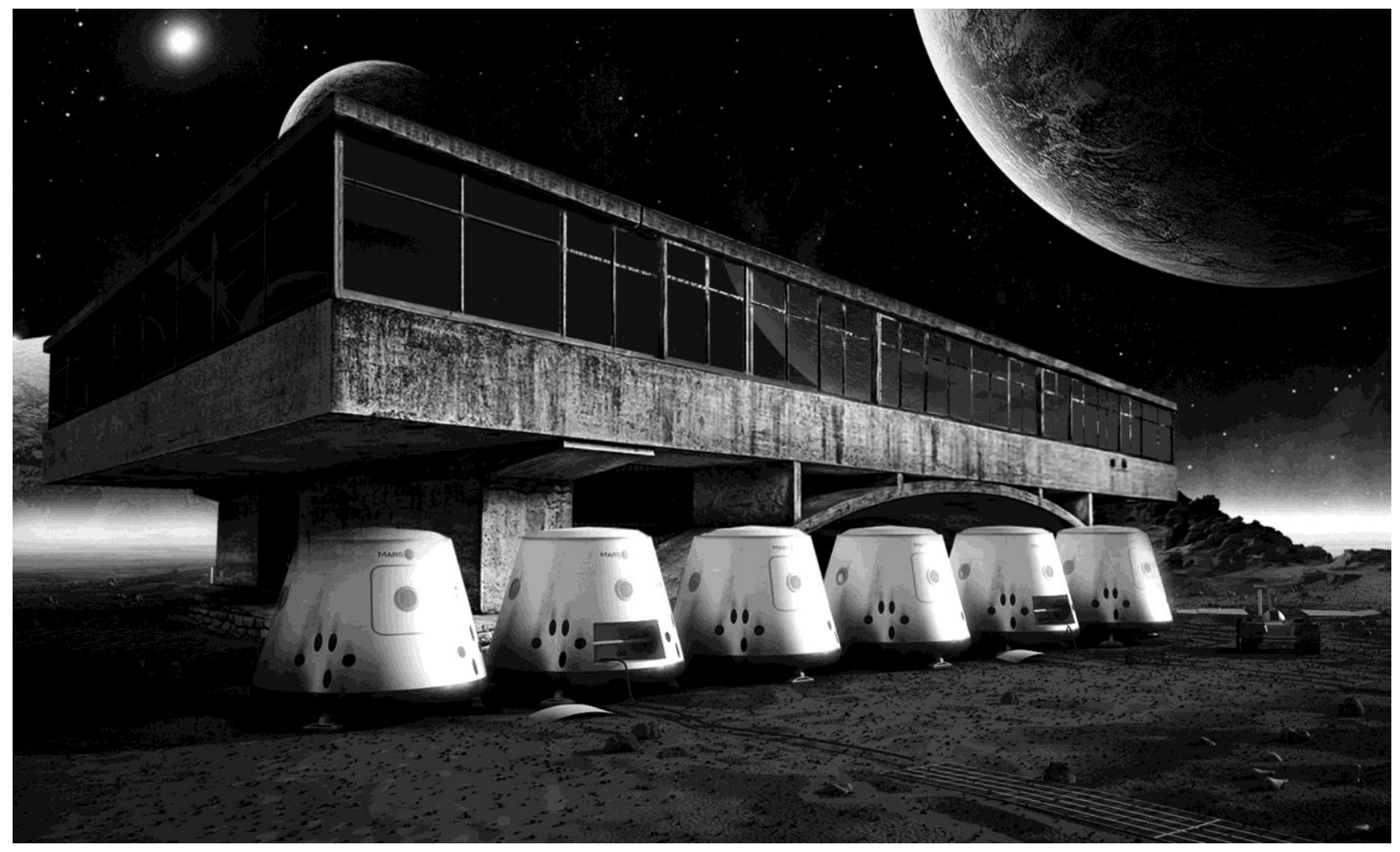

Figura 3. Fotomontaje exterior. Ezequiel Muñoz y Manuela Pozzobón, 2016 


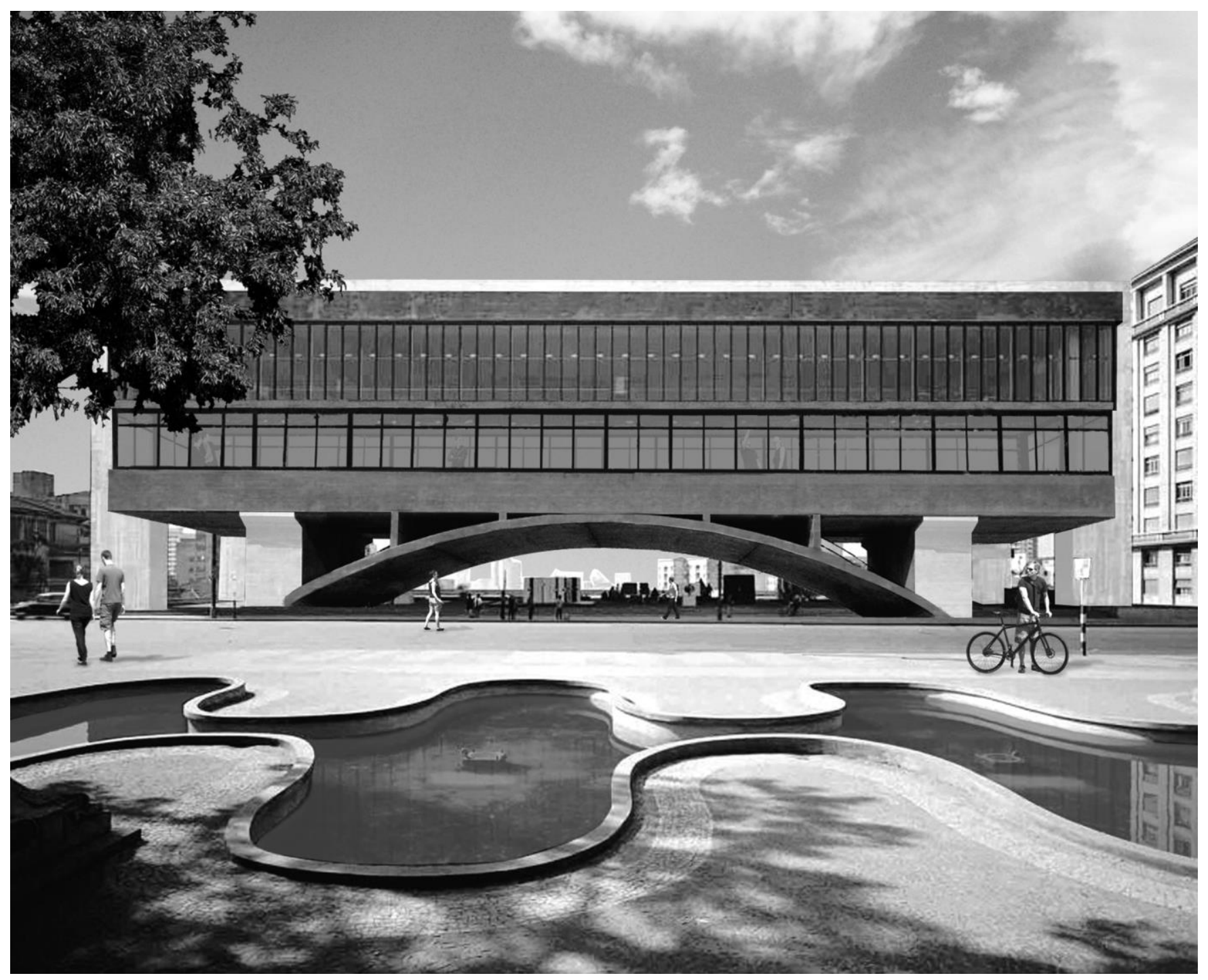

Figura 4. Fotomontaje exterior. Agostina Maschio y Valeria Mangione, 2015

expresivos y comunicacionales complementarios. Ambos aspectos fueron integrados en el planteo inicial de narrativas visuales subyacentes (Mack \& Sklar, 2008), asumidas como recursos didácticos que se resuelven según historias singulares y concisas

Los objetivos generales del curso han sido precisado, por un lado, explorar modos no tradicionales para intervenciones proyectuales de re-contextualización, representación y comunicación de obras de Arquitectura en entornos postdigitales. Por otro, idear y gestionar narrativas visuales, así como desarrollar y optimizar metodologías de estudio y trabajo sobre tratamiento de imagen digital.

Nos centraron en intervenciones sobre la Casa del Puente o Casa del Arroyo. Este edificio emblemático del Movimiento Moderno latinoamericano, es obra del arq. Amancio Williams, representante reconocido del período purista del Movimiento Moderno en Argentina. Fue diseñada y construida en la ciudad de Mar del Plata, durante los años
1943-1946 para residencia familiar veraniega en la ciudad balneraria. La obra está implantada en medio de dos hectáreas con frondosa arboleda. Desarrolló características tecnológicas de vanguardia para cubrir el arroyo de las Chacras, a la manera de un puente (Merro Johnston, 2014). La obra ha sido en declarada de Interés Patrimonial, Cultural y Natural por parte la Municipalidad de General Pueyrredón en 1977, y es también Monumento Histórico Artístico Nacional y Patrimonio Cultural, Histórico, Arquitectónico y Ambiental Prov. Bs As, Argentina. Actualmente sede de un museo municipal Disponible URL https://www.facebook.com/amigoscasa (Ver Fig. 1)

\section{Metodología y técnicas empleadas}

Reconocemos en esta experiencia subyacer la propuesta metodológica del Pensamiento de Diseño (Brown, 2016), a partir de registrar una serie de espacios de acción para desarrollar actividades no necesariamente secuenciales y en un continuo de procedimientos hacia la innovación. Recorrimos así espacios para Inspirar, Idear, Experimentar e 
Implementar, favorecidos por retroalimentaciones, reflexiones y reseñas. Desde este enfoque abordamos el planteo, el análisis y la resolución en momentos de gestión, diseño, producción, comunicación y publicación de bases de datos gráficas arquitectónicas, sustentadas y expresadas como narrativas visuales.

Sin embargo, sin alejarnos de esta perspectiva, nos ha interesado enriquecer a la misma con aportes provenientes de la metodología de Dunne \& Raby (2013, op.cit.), desde el enfoque que presenta el Diseño Especulativo y afín a los ambientes experimentales de los modalidades de trabajo de los MediaLab. En particular, tal propuesta metodológica se encuentra a mitad de camino entre la eficiencia a ultranza y la experiencia estética pura. Consiste en crear y re-crear nuevos objetos producidos a partir de imágenes de distinto tipo (fotos, videos y textos prioritariamente) para insertarlos en escenarios ajenos y extraños al objeto original. Como requisito inicial, requiere de la existencia previa de narrativas visuales con relatos amplios y diversificados que la estructuren. Asimismo alienta el espíritu del diseño participativo, abierto y bordando un límite conceptual que pide la complicidad del público para convertirse en producto, inestablemente acabado. El público en tal circunstancia actúa no sólo como observador/consumidor, sino como usuario/productor a la manera de co-diseñador y prosumidor.

El curso, realizado en modalidad didáctica de Taller, recurrió al uso de plataformas estándares interconectadas, aplicaciones gráficas genéricas y de acceso libre. En esta dirección, abordó la enseñanza de programas estándares sobre tratamiento de la imagen pixelar y vectorial, e incursionó en la exploración de aplicaciones y recursos hipermediales colaborativas interconectadas a la Web para difusión y publicación de información prioritariamente gráfica.

En primer lugar, precisamos el diseño de las tramas narrativas visuales (Morra, 2013). Estas fueron los ejes para argumentar la construcción de los posteriores fotomontajes y piezas comunicacionales en escenarios exteriores e interiores. Al respecto nos basamos en una serie de principios sobre escritura de ficción televisiva (Espenson, 2010), reseñados según creación de mundos y escenarios complejos, protagonismo de información visual, prontitud del discurso, sugerencia antes que elucidación amplia, apreciación de aspectos documentales, dispersión del relato de la pantalla, jerarquización de luz y sombras, sostenimiento especificidad de temáticas, presencia de argumento enérgico para sostener el relato, sustento fiel con la realidad propuesta, posibilidad de variación de las normas fijadas, integración factible de personajes a escenarios, aumento de hipermedialidad, alteración de esquemas (familiares, profesionales, personales) tradicionales, integración con temporalidad del devenir histórico

En segundo lugar, afrontamos la ideación, gestión y diseño de los fotomontajes, apelando a recreación de objetos y paisajes originales en nuevos contextos con reinserción de imágenes existentes de la Casa del Puente, registros de campo y recursos varios fácilmente disponibles en la Web a partir de la inclusión de elementos externos y extemporales. Se realizaron los necesarios ajustes, modificaciones, transformaciones, distorsiones o correcciones, junto al control de proporciones, colores, texturas e iluminaciones. Se incorporaron elementos ajenos para lograr veracidad e hiperrealismo (ver Figs. 2, 3, 4, 5, 6)

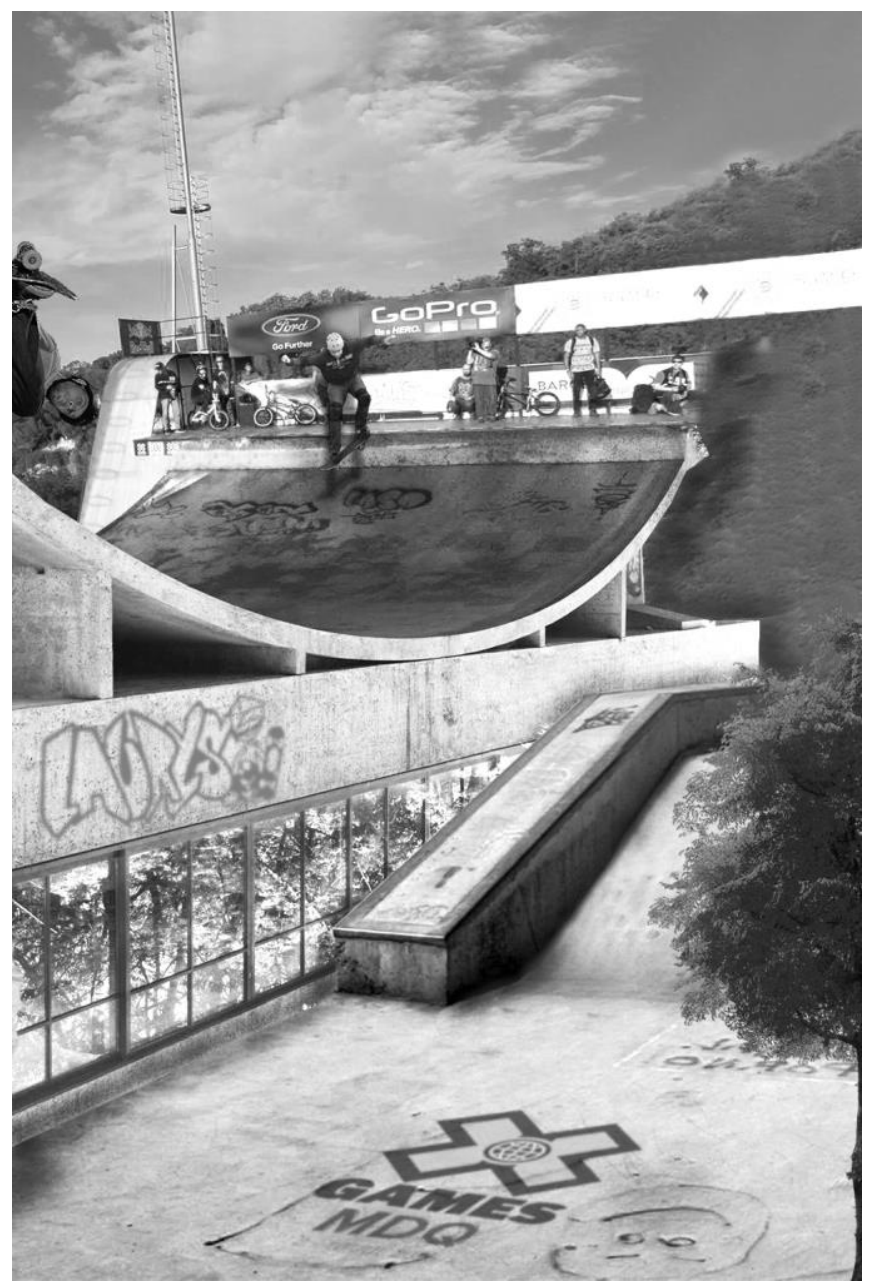

Figura 5. Fotomontaje exterior, Juan Petrecca, 2015

En tercer lugar, la tarea se completó con la ideación y producción de piezas comunicacionales gráficas impresas en diferentes formatos (afiches, posters, catálogos, revistas, etc) y prototipado de tales piezas generados.

Finalmente, en cuarto lugar, se realizaron infografías, presentaciones digitales y diseño de sitios web con aplicaciones gratuitas interconectadas. En cada etapa, reflexionamos de forma crítica las ventajas y limitaciones de tales recursos, y se retroalimentó el proceso.

\section{Resultados}

Analizamos y evaluamos la experiencia desde la perspectiva de los procesos co-creativos en entornos post-digitales. Ampliamos y reformulamos indicadores de creatividad tradicionales (Vessey \& Mumford, 2012; Boden, 2011) agrupadas en categorías sobre indicadores de unicidad, originalidad, fluidez y alta frecuencia ideas; de planteo, desarrollo, profundización y fin producciones; de flexibilidad, divergencia y convergencia, reestructuración y 
reconstrucción problemas, conjunción, análisis y síntesis; de iniciativa, independencia, motivación, empatía y sensibilidad ante problemas; de racionalización, innovación y transferencia.

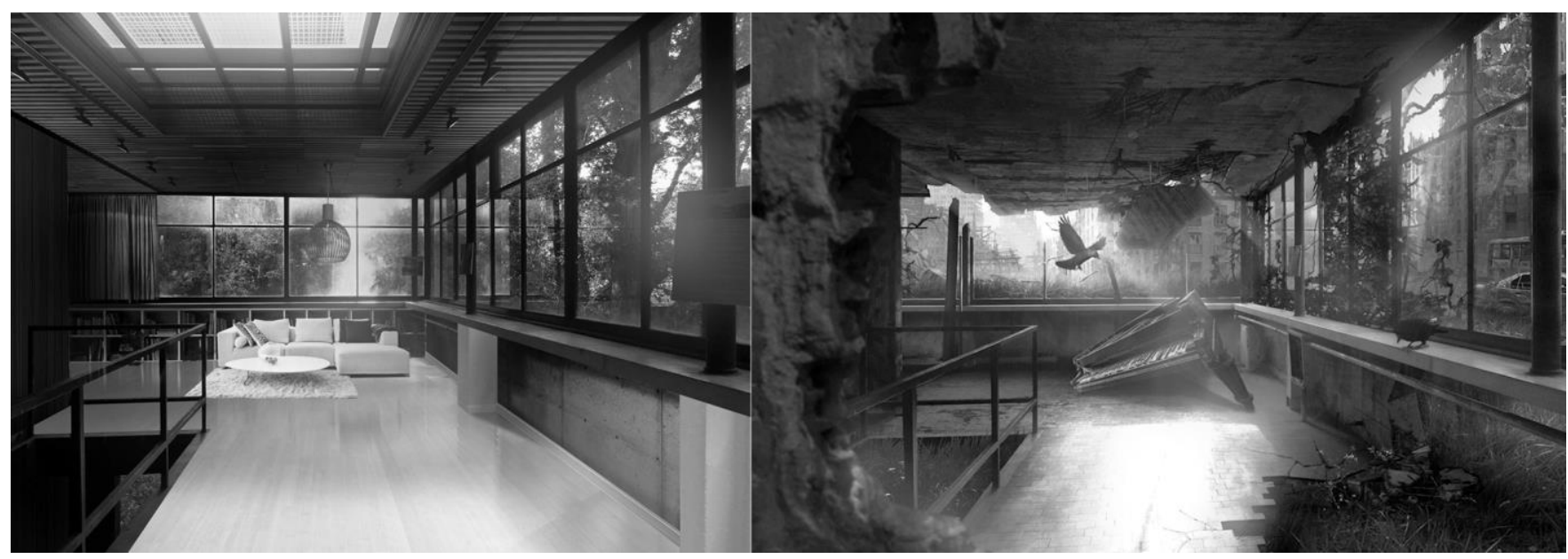

Figura 6. Fotomontajes interiores. Massimiliano Depalo, 2015

Observamos que la experiencia tuvo resultados mayoritariamente positivos. Los mismos se potenciaron con producciones singulares que facilitaban experiencias estéticas y lúdicas, resultando enriquecedoras, interactivas y gratificantes, tanto en la construcción de las narrativas, los diversos fotomontajes como en el diseño de las piezas comunicacionales. En tales circunstancias, registramos que mayoritariamente fueron estímulos positivos en el desarrollo de la tarea.

En particular, las líneas narrativas suministraron claridad y coherencia al desarrollo de la trama y las producciones resultantes; generaron interés y desarrollo comprometido de la tarea; ordenaron la secuencialidad desde instancias iniciales, de desarrollo y de desenlace de las situaciones propuestas; valoraron efectivamente la fuerza evocativa y expresiva de la imagen en la selección de escenarios, escenas, objetos, personajes. Verificamos resultados originales próximas a intervenciones de naturaleza transmedial, cuando se mixturaban narrativas inspiradas en reelaboración de argumentos de libros, películas, series o comics. Los mismos resultaron en activadores visuales y textuales que movilizaron gestión, producción, evaluación y almacenamiento de la información y aportaron a la construcción de sentido.

También registramos competencias híbridas e-skills en los estudiantes que han inducido instancias de co-creación e innovación en etapas de aprendizaje basado en proyectos y en instancias de aprendizaje permanente.

Al respecto, detectamos instancias de auto-aprendizaje, autoevaluación, evaluación entre pares y retroalimentación reflexiva en forma constante. En las mismas los estudiantes han explorado, indagado el tema, trabajado en equipo demostrando fluidez en la comunicación y la organización, responsabilidad individual y grupal, interacción colaborativa (para conectarse, complacerse, escuchar, establecer vínculos y encontrar otras ideas y estímulos esencialmente diferentes) e integración a redes.

\section{Conclusiones y discusiones}

Desde la perspectiva del Diseño Especulativo, en esta experiencia pedagógica en entornos post-digitales, nos ha interesado transformar los objetos/resultados en proyectos como procesos profundos y complejos, que tal vez contengan nuevos objetos, pero ideados y producidos desde los diversas espacios de acción propios del Pensamiento de Diseño. En tal proceso reconocemos el soporte del relato de la narrativas visual que lo sostiene y le da sentido en la producción.

Al respecto, coincidimos con María Acaso (2015), al considerar primero, que tales proyectos nos hacen reflexionar sobre la visualidad y los modos de co-creación, asumiendo que la ideación y la construcción de las mismas son procesos sumamente densos donde transcurren numeroso niveles de significación colectivos. Segundo, en la necesidad de utilizar la tecnología post-digital al servicio del conocimiento, asumiendo que deviene en un requisito secundario a la idea, siendo ésta última el comienzo de la acción y la inspiración para generar colaborativamente, o sea co-crear, nuevos proyectos. Tercero, estos procesos de cocreación pueden ser soportados por teorías refutables y experiencias i-replicables en consonancia con la idea de descentrar al objeto de los procesos y desplazarlos fuera de la acción; tal circunstancia implica el abandono de la réplica desde la simplicidad, abordando las cuestiones simples como problemas complejos en vez de abordar los problemas complejos de manera simple.

En particular y como tema de discusión, reflexionamos sobre estas experiencias que rozan los límites expresivos y creativos disciplinares en la carrera de Arquitectura y están próximos al concepto transdisciplinar del Art Thinking. Compartimos con Luis Camnitzer (2015) la proposición, entre otras cuestiones, de entender a la enseñanza y en nuestro caso lo hacemos extensivo a la enseñanza de prácticas proyectuales y medios electrónicos, como una cultura compleja de aprendizaje en sí misma. O sea como una metodología de creación de conocimiento para cualquier 
asignatura y no como la construcción de una sucesión de aprendizajes de técnicas e instrumentos.

Por último, la experiencia nos ha servido para retomar aciertos, reflexionar críticamente en los desaciertos y entenderla como fuente de inspiración para continuar recreando otros proyectos en la actualidad en la misma dirección.

De forma complementaria, con la producción de ambos cuatrimestres se ha planificado realizar una muestra junto con la Asociación Amigos de la Casa del Puente en la ciudad de Mar del Plata.

\section{Agradecimientos}

El presente escrito se encuadra en estudios del proyecto 15/B277 SCTyC UNMdP 2014-2015, actualmente continuado en el proyecto 15/B310 SCTyC UNMdP 2016-2017, Grupo EMIDA (CEAC) CIPADI FAUD, ambos con dirección de Diana Rodríguez Barros.

El equipo docente ha estado integrado por las diseñadoras gráficas María Mandagarán e Inés Hernández y contó con la colaboración de la estudiante avanzada Tamara Conforti.

\section{Referencias bibliográficas citadas}

Acaso, M. (2015). Desplazar al objeto: la importancia de pasar de los instrumentos a la cultura del aprendizaje en el contexto del arte + educación. En María Acaso. Disponible http://tinyurl.com/hlm5llz

Boden, M. (2011). The creative Mind. Myths and Mechanisms. Barcelona: Ed. Gedisa.

Brown, T. (2016). The Next Big Thing in Design. En Design Thinking. http://designthinking.ideo.com/?p=1451

- (2009). Change by design: how Design Thinking transforms organizations and inspires innovation. New York: Harper Collins.

Camnitzer, L. (2015) Thinking about art Thinking. En e-flux journal 56th Venice Biennale. Disponible http://supercommunity.e-flux.com/texts/thinking-about-artthinking/

Cramer, F. (2014). What is Post-digital? En APRJA A PeerReviewed Journal About Post-digital Research. Vol.3, Issue 1. Disponible http://www.aprja.net/?p=1318

Dunne, A. \& Raby, F. (2013). Speculative everything: design, fiction and social dreaming. The MIT Press

Espenson, J. (2010). Chapter 5. Playing Hard to 'Get' - How to Write Cult TV. En Abbott, S. edit. The Cult TV Book . London: I.B.Tauris \& Co. Pp 45, 54

Ibars, R.; Ma, L. (2013). Critic Design \& Speculative Design. En "Jinhuà, de la imagen a la ciencia, de la ciencia al diseño” Roger lbars y Lisa Ma. En Co-creatingculture.
Disponible http://co-creating-cultures.com/es/?tag=rogeribars

Jenkins, H. (2012). Transmedia storytelling. Moving characters from books to films to video games can make them stronger and more compelling. En MIT Technology Review. January 15. Disponible http://tinyurl.com/k3bbveg

Lima, M. (2011). Visual complexity: mapping patterns of information. New York: Princeton Architectural Press: Pp. 232-243

Mack, A. \& Sklar, A. (2008) Greater good. En Newdesign magazine. Issue 63. Warwickshire UK: Media Culture. Pp.20-22.

Disponible http://www.ideo.com/images/uploads/news/pdfs/NewDesi gnUK_63_08.pdf

Merro Johnston, D. (2014). La casa sobre el arroyo. Amancio Williams en Argentina. Buenos Aires: Ediciones 1:100.

Morra, S. (2013). Great digital storytelling. En Educational uses of digital storytelling. University of Houston Education. Disponible http://tinyurl.com/p6hophg

Pardo Kuklinky, H. (2010) Geekonomía. Un radar para producir en el postdigitalismo. Barcelona: Colección Transmedia XXI Universitat Barcelona.

Rodríguez Barros, D.; Mandagarán, M.; Nigro, P.; Hernández, M.I. (2015). Pensamiento de Diseño, Narrativas Visuales y Creatividad. Un caso de prácticas didácticas disruptivas en entorno postdigital. XIX Congresso da Sociedade Ibero-americana de Gráfica Digital 2015, En São Paulo: Blucher Design Proceedings, Volume 2, 2015, Pages 618-622, Florianópolis, Brasil, Universidade Federal de Santa Catarina. DOI http://dx.doi.org/10.1016/despro-sigradi2015-100381

Rodríguez Barros, D.; Mandagarán, M. (2014). Pensamiento de diseño y construcción de narrativas visuales. Caso de prácticas didácticas disruptivas en entornos postdigitales. En García Amen, F. editor. Libro de ponencias XVIII Congreso SIGraDi "Diseño Basado en Conocimiento". Montevideo: SIGraDI \& Universidad de la República. Pp. 195-199

Sander, L. \& Simons, G. (2009). A social visión for value cocreation in Design. En TIM Technology Innovation Management Review. Disponible http://timreview.ca/article/310

Scolari, C. (2013). Narrativas transmedias, más allá de la pantalla: los parques de atracciones. En Hipermediaciones. Disponible http://tinyurl.com/kwm7qrq

Vessey, W. \& Mumford, M. (2012). Heuristics as a basis for assessing creative potential: Measures, methods, and contingencies. En Creativity Research Journal vol. 24 issue 1 . Measuring creativity. Pp. 41-54. Disponible http://www.tandfonline.com/doi/full/10.1080/10400419.20 12.652928\#.VWMIYqbidRk 